\title{
GPS Collar Sampling Frequency: Effects on Measures of Resource Use
}

\author{
Dustin D. Johnson ${ }^{1}$ and David C. Ganskopp ${ }^{2}$
}

Authors are ${ }^{1}$ Assistant Professor, Department of Rangeland Ecology and Management, Harney County Extension Office, Oregon State University, 450 North Buena Vista, Burns, OR 97720, USA; and ${ }^{2}$ Rangeland Scientist, United States Department of Agriculture-Agricultural Research Service, Eastern Oregon Agricultural Research Center, 67826-A Hwy 205, Burns, OR 97720, USA.

\begin{abstract}
A challenge in animal behavior studies using Global Positioning System (GPS) collars is selecting a sampling frequency to accomplish desired goals. High data resolution (i.e., frequent sampling) is appealing, because it maximizes behavioral information garnered. Extended sampling might be needed, however, to describe long term behaviors or seasonal dynamics. Because tradeoffs exist between high data resolution and sampling duration, we evaluated the effects of variable GPS sampling intervals on proportions of pastures used by cattle and distance traveled per day. This was accomplished with GPS collars configured to record cattle positions every $5 \mathrm{~min}$ for $15 \mathrm{~d}$ in three 829-864-ha pastures. Data were iteratively reduced to simulate increasingly longer GPS recording intervals from once every $10 \mathrm{~min}$ up to once daily. Two techniques were used to measure the percentage of pastures accessed by cattle. The first counted only pixels containing GPS coordinates. The second counted pixels containing coordinates and/or traversed by lines between vertices. Expansion of GPS recording intervals decreased $(P<0.01)$ estimates of the proportion of pastures visited by cattle with rates of decline best fit by exponential decay functions for both line and point techniques $\left(R^{2}=0.93\right.$ and 0.97 , respectively). Spatial errors accompanying less frequent sampling intervals, however, were extremely large with the line technique and misrepresented areas visited by cattle. Expansion of GPS sampling intervals decreased $(P<0.001)$ distance traveled per day by cattle about $10 \%$ with each iteration. If travel corridors or accurate assessments of resources accessed are of critical concern, then longer GPS integration intervals should be avoided because they propagate flawed spatial interpretations. Similarly, if accurate measures of travel distances are critical, we suggest using a relatively frequent GPS recording interval.
\end{abstract}

\section{Resumen}

La selección adecuada de la frecuencia de muestreo para lograr las metas deseadas es un reto en los estudios de comportamiento animal cuando se usan collares del sistema global de posición (GPS). Generalmente se busca obtener un número extenso de datos (es decir muestreo frecuente) porque proporciona información más acertada sobre comportamiento. Un muestreo prolongado puede ser necesario dado que el GPS describe el comportamiento a largo plazo o las dinámicas estaciónales. Debido a que existe una relación entre la alta resolución de los datos y la duración del muestreo, se evaluó el efecto del intervalo de muestreos variables del GPS en proporciones de potreros usados por el ganado y la distancia caminada por día. Con este fin, se utilizaron los collares del GPS configurados para registrar la posición del ganado cada 5 minutos durante 15 días en tres potreros de 829-864 ha. La información fue interactivamente reducida para simular intervalos cada vez más largos en la grabación del GPS usando intervalos desde 10 minutos hasta una vez al día. Dos técnicas fueron utilizadas para medir el porcentaje del potrero al cual el ganado tuvo acceso. El primer conteo incluyó únicamente pixeles que contienen coordenadas del GPS. El segundo contó los pixeles que contenían coordenadas y/o líneas a través de los vértices. Al aumentar la extensión de los intervalos de la grabación del GPS se disminuyó $(P<0.01)$ la estimación de la proporción del potrero visitado por el ganado con tasas de reducción que se explicaron mejor usando tasas de reducción de la función exponencial por las técnicas de línea y de punto $\left(R^{2}=0.93\right.$ y 0.97 , respectivamente). Los errores espaciales producidos por los intervalos de muestreo menos frecuentes, fueron extremadamente grandes con la técnica de línea y no representativas de las áreas visitadas por el ganado. La extensión de los intervalos de muestreo del GPS disminuyó $(P<0.001)$ cerca de $10 \%$ la distancia caminada por el ganado al día. Si el recorrido caminado o la acertada evaluación de los recursos evaluados son de preocupación crítica, entonces los intervalos más largos de la integración del GPS deben evitarse porque conducen a interpretaciones espaciales equivocadas. En contraste, si las medidas exactas de los recorridos son críticas, entonces sugerimos un intervalo relativamente frecuente de la grabación del GPS.

Key Words: cattle, distance traveled, GPS collars, grazing behavior, sampling interval

Eastern Oregon Agricultural Research Center, including the Burns and Union Stations, is jointly operated by the Oregon Agricultural Experiment Station of Oregon State University, Corvallis, Oregon and the United States Dept of Agriculture-Agricultural Research Service.

Mention of trade or proprietary names is for information only and does not convey endorsement of one product over another.

Correspondence: Dave Ganskopp, United States Dept of Agriculture, Eastern Oregon Agricultural Research Center, Burns, OR 97720, USA. Email: david.ganskopp@oregonstate.edu

Manuscript received 10 May 2007; manuscript accepted 16 November 2007.

\section{INTRODUCTION}

Global Positioning System (GPS) technology is a relatively recent development (e.g., Rodgers and Anson 1994; Rodgers et al. 1996; Agouridis et al. 2004) for monitoring travel (Brosh et al. 2006), activity (Ungar et al. 2005), and resource use by medium- to large-sized animals (Mourão and Medri 2002; 
Bailey et al. 2006). GPS receivers in a lightweight collar or harness can be deployed for extended periods with little effect on behavior. Units derive coordinates from an internal receiver tracking an array of 24 earth-orbiting satellites. Coordinates are stored in onboard memory for later retrieval or relayed via phone or radio to other memory.

One challenge in animal studies with GPS collars is selecting suitable sampling intervals. Intuitively, one desires high data resolution (i.e., short sampling intervals) to maximize behavioral information garnered. Considerations, however, involve tradeoffs between data resolution, battery life, memory constraints, and the question of interest.

If an animal randomly visited areas within its habitat, measures of resource use should mirror an exponential decay function as sampling intervals are progressively extended (Marquardt 1963). However, many animals are "central place foragers" (Stuth 1991). They repeatedly revisit favored feeding sites, nearby water, and limited escape terrain or resting areas. Given their nonrandom use of the landscape, measures of habitat use and travels might or might not track expectations with increasingly extended sampling intervals. The objective of this study was to quantify the effects of different GPS sampling intervals on estimates of distance traveled and the percent of pasture area visited by cattle. This was accomplished by extracting subsets of less frequent recording regimes from GPS data originally acquired at 5 -min intervals.

\section{MATERIALS AND METHODS}

\section{Study Site}

Research occurred simultaneously in three pastures (829864 ha) on the Northern Great Basin Experimental Range (lat $43^{\circ} 29^{\prime} \mathrm{N}$, long $119^{\circ} 43^{\prime} \mathrm{W}$ [WGS-1984 datum]; elevation 1400-1674 m), $52 \mathrm{~km}$ west of Burns, Oregon. Mean annual temperature is $7.6^{\circ} \mathrm{C}$ with recorded extremes of $-29^{\circ} \mathrm{C}$ and $42^{\circ} \mathrm{C}$. Mean annual precipitation is $289 \mathrm{~mm}$ with about $60 \%$ being snow.

Vegetation is characterized by a sparse western juniper (Juniperus occidentalis subsp. occidentalis Hook.) overstory and a shrub layer dominated by Wyoming big sagebrush (Artemisia tridentata subsp. wyomingensis Beetle \& Young), mountain big sagebrush (Artemisia tridentata subsp. vaseyana [Rydb.] Beetle), or low sagebrush (Artemisia arbuscula Nutt.). Dominant grasses include bluebunch wheatgrass (Pseudoroegneria spicata [Pursh] A. Löve), Idaho fescue (Festuca idahoensis Elmer), or Sandberg's bluegrass (Poa secunda J. Presl), depending on locale. Just prior to our study in 2004, mean herbaceous standing crop averaged $357 \mathrm{~kg} \cdot \mathrm{ha}^{-1} \pm 3.9 \mathrm{SE}$ among the three pastures.

\section{GPS Collar Sampling Protocol}

GPS collars supported an 8-channel receiver (Lotek ${ }^{\circledR}$ GPS2200LR; Lotek Engineering Inc., Newmarket, Ontario, Canada) capable of simultaneously tracking $\leq 8$ satellites. Records were written to onboard, nonvolatile random access memory retaining a maximum of 5028 differentially correctable coordinates. Each record included collar number, date, time, longitude and latitude, elevation, a dilution of precision value (an index of satellite geometry reflecting position accuracy), a 2- or 3-dimension fix status (2-dimension records are derived from a minimum of three satellites with no elevation estimate, whereas 3 -dimension records use $\geq 4$ satellites and generate an elevation measure), ambient air temperature, and satellite information used for differential correction.

On 12 June 2004, 60 cow-calf pairs were randomly divided among three groups. Four cows from each group were randomly selected, fitted with GPS collars, and groups randomly dispersed among the three pastures. Collars were programmed to record data every $5 \mathrm{~min}$ beginning at 0000 hours 15 June 2004 and stopping $15 \mathrm{~d}$ later at 2355 hours on 29 June 2004. Twelve records were expected each hour, 288 records $\cdot \mathrm{d}^{-1}, 4320$ records $\cdot$ collar $^{-1}$ over the 15 -d trial, and a total of 51840 records across all 12 collars.

\section{GPS Collar Data}

Collars were retrieved 30 June 2004. One collar failed completely, and a second stopped operating after 3932 records. Including the partially functioning collar, we acquired $90.9 \%$ of our targeted data.

Coordinates were differentially corrected with N4 v.1.1895 software (Lotek Engineering). Base station files were acquired from a US Forest Service/Bureau of Land Management station near Hines, Oregon, approximately $50 \mathrm{~km}$ east of our pastures (http://www.fs.fed.us/database/gps/burns.htm). Differential correction processes occasionally failed if roving or base station units viewed slightly different satellite arrays. Among the 11 functioning collars, differential correction failure averaged $4.9 \pm 1.1 \mathrm{SE}$ observations $\cdot$ collar $^{-1}$, with uncorrected coordinates substituted in all instances. Latitude and longitude were subsequently converted to Universal Transverse Mercator (UTM 11N-WGS-1984) coordinates with Idrisi32 v.32.22 software (Clark Labs, The Idrisi Project, Worcester, MA).

\section{Resource Use}

Custom QBasic software was compiled to iteratively omit records from our original 5-min sampling interval files to reflect increasingly longer recording intervals. For example, if every other record beginning with the second was omitted, the resulting file supported half as many observations $(n=2160)$ and mirrored data acquired at a 10-min interval. Analyzed intervals included $5,10,20,40,80,160,360,480,720$, and $1440 \mathrm{~min}$ (all evenly divisible into $24 \mathrm{~h}$ ). The last interval (1440 min) was a once-daily sampling with each record acquired at midnight. With 10 files generated from each of the 11 functioning GPS collars, each evaluation processed a total of 110 data sets.

UTM coordinates for each of the files were imported into Global Mapper v.6.06 (Global Mapper Software LLC, Olathe, KS) using two methods. The first treated each record as a standalone coordinate or point. The second treated coordinates as vertices and rendered connecting lines between successive records. Line vertices were synonymous with points occupied by cattle, and the straight-line paths between vertices captured potential travel routes. Thus, renderings of habitat use should be slightly inflated with the line method.

Point and line files were imported into Idrisi32 v.32.22 and converted to rasterized Boolean images of the pastures with each 


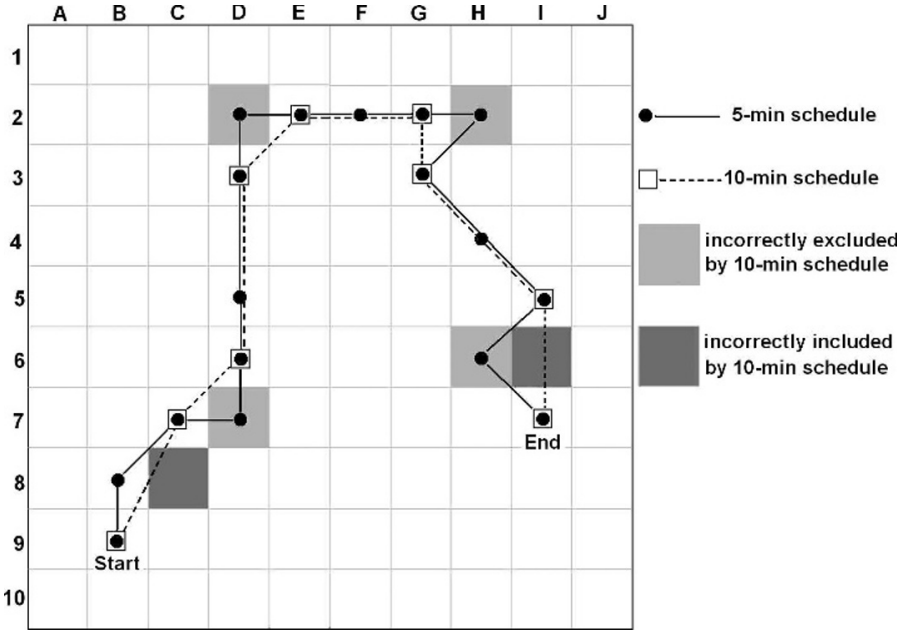

Figure 1. A schematic of travel corridors among 1-ha pixels assuming straight-line pathways between Global Positioning System records at 5and 10-min intervals. The objective is to illustrate potential spatial errors where longer sampling intervals might incorrectly exclude areas actually traversed or mistakenly include areas not truly visited by an animal.

pixel being 1 ha. The resulting 110-point images had a value of 1 in any pixel occupied by a GPS coordinate and a zero for unoccupied pixels. With line files, pixels containing a GPS coordinate or traversed by a line between coordinates were given a value of 1 . Pixels not visited or traversed contained a zero.

Frequency histograms of values in rasterized images were generated for each collar and recording interval and for both point and line images. The number of pixels captured during the 15 -d grazing period was divided by the number of pixels within a pasture to index the proportion of pasture visited and/ or traversed by each cow.

Figure 1 schematically illustrates potential spatial differences when comparing 5- and 10-min GPS sampling regimes. The obvious difference for the two schedules with point files is the capture of 17 and 9 pixels, respectively, with the disparity simply a function of sample size. With the use of line files spanning potential travel corridors between coordinates, the 5 and 10-min schedules in Figure 1 captured 18 and 15 pixels, respectively. Three issues develop with the use of lines files and extended sampling intervals. These involve mistakenly omitted or included pixels, and they are discussed below.

Addition and subtraction of the various Boolean line and point images with the Idrisi image calculator (Clark Labs, The Idrisi Project) were used to determine the number of pixels occurring in the following categories. First were pixels mistakenly included by a coarser scale that were not truly occupied or traversed at the more frequent 5 -min recording interval. These are implied shortcuts captured by the $10-\mathrm{min}$ interval and include pixels 6-I and 8-C in Figure 1.

Second is an issue of mistakenly omitted pixels with a coarse schedule. These areas were indeed occupied at the smallest interval but bypassed or omitted by the larger. The four omitted examples in Figure 1 at the 10 -min interval include pixels 2-D, 2-H, 6-H, and 7-D. We summed counts of mistakenly included and mistakenly excluded pixels in this study and classified their sum as "spatial error."

Third, as sampling intervals are progressively extended, more area is captured solely by lines crossing pixels between distant coordinates. For 10-min data in Figure 1, pixels 8-C, 5-D, 4-D, 2-F, 4-H, and 6-I are examples. Portions of these lines or spans might or might not reflect reality, depending on whether they were truly visited by cattle at the finest sampling interval. Three 10-min interspace pixels that were indeed truly occupied at the 5-min scale include 5-D, 2-F, and 4-H (Fig. 1). Pixel 4-D is an example of an area captured by a line and not definitively occupied at even the finest scale (Fig. 1). In this discussion we pooled counts of the shortcut pixels, identified in our first instance above (6-I and 8-C, Fig. 1), and pixel 4-D identified in our last effort here, into a category labeled "interpolated" data.

\section{Distances Traveled}

Distance between successive UTM coordinates of point files were derived in spreadsheets to estimate average distance traveled by cattle per day. Because a straight-line path was assumed between successive coordinates, and animals often meander as they walk, accumulated distances are likely underestimated by even our most frequent (5-min) GPS sampling interval.

\section{Statistical Analyses}

Because cattle in a given pasture are not always independent entities, the experimental unit in this study was a pasture. The three or four collared cattle (depending on the number of functioning GPS collars) in each pasture were treated as subsamples. Thus, data were averaged among cattle within a pasture and the resulting means applied in analyses.

Regression models were fit relating mean daily distance cattle traveled and percentages of pastures visited by cattle over the 15-d trial (dependent variables) with increasing GPS recording intervals (5- to 1440-min) as the independent variable. Because scattergrams suggested a curvilinear function was most appropriate, polynomial and exponential decay models were applied and models exhibiting the highest coefficients of determination $\left(R^{2}\right)$ retained.

Last, because cattle in arid regions are often "central place foragers" (Stuth 1991), we tested the hypothesis that a regularly scheduled but infrequent GPS sampling interval (i.e., 1440 min or once daily) might bias one's interpretations about an animal's relationship to a limited or required resource such as water. We used the twice daily GPS sampling regime (720 min), with daily records acquired at midnight and noon, to test this hypothesis. A paired $t$ test compared distances of cattle from water at midnight and noon with expectations that midday observations would find animals nearer water. Statistical significance in all analyses was assumed at $P \leq 0.05$. In all text and tables, values following \pm symbols are standard errors (SE) of the mean $(n=3)$.

\section{RESULTS}

\section{GPS Sampling Interval Effects on Percent of Pasture Visited}

With a 5-min GPS schedule over a 15-d interval, cattle were found in $286.6 \pm 4.6$ pixels $\cdot$ pasture $^{-1}$ with the point technique and $307.0 \pm 1.1$ pixels $\cdot$ pasture $^{-1}$ with the line method. Pastures averaged $846.6 \pm 9.1$ pixels, so cattle visited approximately $33.8 \pm 0.5 \%$ and $36.3 \pm 0.3 \%$ of their pastures, respectively, with the point and line methods (Fig. 2). 


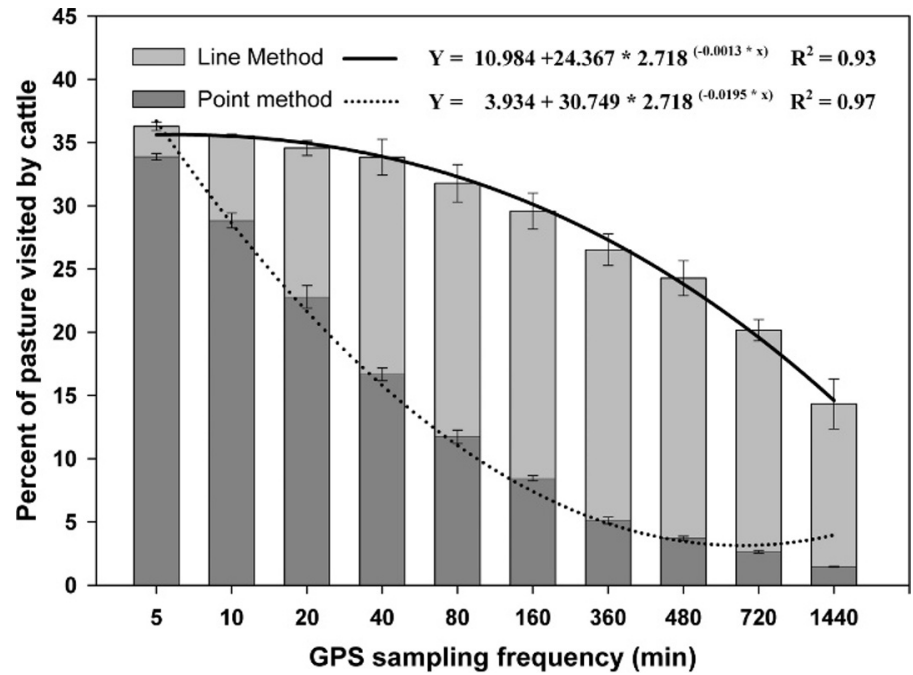

Figure 2. The percent of 1-ha pixels \pm 1 SE within three $800+$ ha pastures visited by Global Positioning System (GPS)-collared cattle as measured by two mapping techniques and a variety of GPS sampling frequencies from once every 5 min (288 positions daily) to once every 1440 min (once daily) gathered over a 15-d grazing trial in June 2004 on the Northern Great Basin Experimental Range near Burns, Oregon. The first method (point technique) included only pixels containing GPS coordinates. To also identify potential travel corridors, the second method (line technique) captured pixels occupied by GPS coordinates and/or traversed by a line connecting adjacent coordinates. Plotted lines depict modeled estimates of the percent of pasture visited by cattle with three-parameter exponential decay functions for both the line and point methods.

As GPS sampling intervals were progressively expanded, estimates of the percent of pasture visited by cattle declined with both methods (Fig. 2). The rate of information loss with both techniques was best fit $(P<0.001)$ with three-parameter exponential decay functions (Fig. 2). As GPS recording intervals expanded, point files exhibited a more rapid decay of estimated pasture use than the line method. Data collected at 40-min intervals with point files suggested cattle accessed only $16.6 \pm 0.5 \%$ of the pasture, about a $50 \%$ decline in estimated use compared to the 5-min regime. The same 5- to 40-min GPS recording interval expansion with the line technique, implied cattle visited $33.8 \pm 1.5 \%$ of the pastures, an implied information decay of about $7 \%$.

If an animal were to walk a perfectly straight line for a given period, any GPS sampling regime and assay using the line technique would capture an identical set of pixels. If that was the case with our cattle, each of the extended GPS recording intervals would have traversed the same 307 pixels captured by our 5-min recording interval. Because cattle do not follow a straight line, a breakdown of captured and omitted data with the line technique into its component parts is presented in Figure 3. Spatial error increased rapidly with the line method as GPS recording intervals were extended (Fig. 3). With the 5-min recording interval, $93 \pm 1.5 \%$ of the pixels identified by the line method contained actual GPS coordinates. Thus, unoccupied interspaces between coordinates constituted the balance $(7 \pm 1.2 \%)$. Again, because we can not verify that cattle actually occupied sites crossed solely by lines, we called these

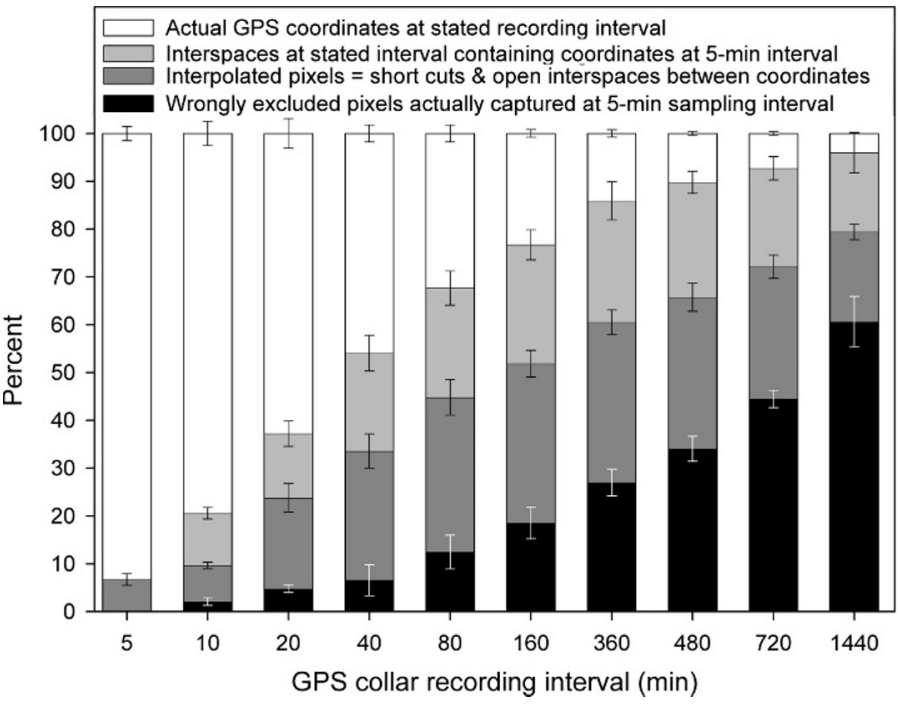

Figure 3. A partitioning of data, captured by the line technique, into valid and incorrectly included and excluded 1-ha pixels potentially visited by Global Positioning System (GPS)-collared cattle in three $800+$ ha pastures over a 15-d grazing trial in June 2004 on the Northern Great Basin Experimental Range near Burns, Oregon, and analyzed at a variety of GPS sampling intervals ranging from once every 5 min to once every $1440 \mathrm{~min}$. Occupied areas were captured with the line technique that identifies all pixels containing GPS coordinates or traversed by a straight line between successive coordinates. Error bars topping each component are $\pm 1 \mathrm{SE}$.

areas "interpolated pixels" in Figure 3, and one can argue for or against including such data in analyses.

With GPS recording intervals $\geq 10 \mathrm{~min}$, however, two more components were partitioned from the data. These were pixels mistakenly excluded at the longer interval, but actually visited by cattle using the 5-min regime, and interspaces between coordinates at the stated sampling interval that were indeed visited by cattle with the 5 -min recording interval. Thus, for GPS recording intervals $\geq 10 \mathrm{~min}$ in Figure 3 , the two uppermost components of a bar are valid or verifiable data. Excluding the initial 5-min sampling regime, the two lowermost components of each bar (interpolated or wrongly excluded pixels) cannot be verified unless one indeed has the luxury of finer-scale data. Again, we called the pooled contribution of the two lowermost components in Figure 3 "spatial error." The cost of expanding the GPS recording interval from 5 to 10 min introduced about $11 \%$ spatial error into estimates of pasture use. With a twicedaily recording interval $(720 \mathrm{~min})$ and the line techniques, $72 \%$ of our data was spatial error (Fig. 3). Interpolated pixels, consisting of shortcuts and open interspaces between coordinates, constituted the bulk of spatial error for GPS recording regimes from 10- to the 360-min interval. With GPS intervals of 480,720 , and $1440 \mathrm{~min}$, wrongly excluded pixels made up the bulk of spatial error.

\section{GPS Recording Interval Effects on Estimates of Distance Traveled}

Successive expansion of GPS recording intervals significantly reduced estimates of daily distance traveled by cattle with the relationship best fit $(P<0.01)$ with a three-parameter exponen- 


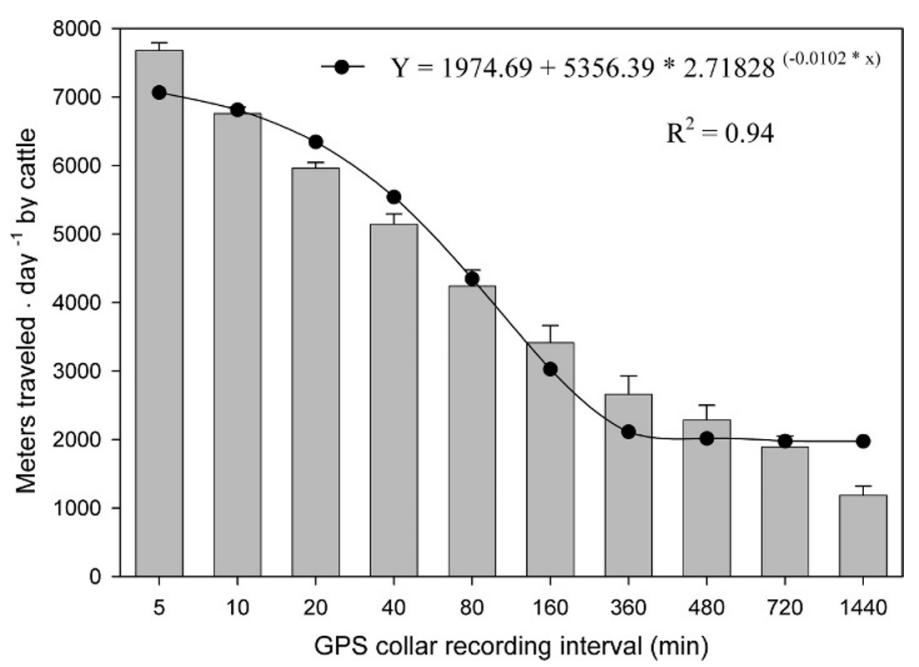

Figure 4. Bars represent estimates of distance \pm 1 SE traveled per day by Global Positioning System (GPS)-collared cattle grazing in three $800+$ ha pastures on the Northern Great Basin Experimental Range near Burns, Oregon, in June 2004 as evaluated with several GPS recording intervals ranging from once every 5 min to once every $1440 \mathrm{~min}$. Distances are straight line measures between successive coordinates. The plotted line depicts predicted values of distance traveled per day by cattle as estimated by a three- parameter exponential decay function.

tial decay function (Fig. 3). Increasing the integration interval from 5 to $10 \mathrm{~min}$ decreased estimated travel from $7680 \pm 115$ $\mathrm{m} \cdot$ day $^{-1}$ to $6759 \pm 94 \mathrm{~m} \cdot$ day $^{-1}$ or about $12 \%$. Slightly more than a $50 \%$ decrease $(4267 \mathrm{~m})$ in estimated travel occurred as the GPS recording interval was expanded to $160 \mathrm{~min}$.

\section{Infrequent GPS Sampling Intervals and Proximity of Cattle to Water}

The twice daily (720 min) GPS recording interval was used to test the hypothesis that infrequent sampling regimes might bias one's interpretations about an animal's relationship to a critical resource like water. Coordinates acquired at midnight found cattle approximately $1166 \pm 143 \mathrm{~m}$ from water and midday coordinates placed them $342 \mathrm{~m}$ closer $(P=0.046)$ at a distance of $824 \pm 148 \mathrm{~m}$. The average distance for cattle from water with our 5-min regime was $1019 \pm 121 \mathrm{~m}$.

\section{DISCUSSION}

Decisions regarding the resolution and analyses of GPS collar data have profound consequences on the quantity and accuracy of information garnered in animal behavior studies. With expanded GPS sampling intervals, we progressively underestimated pasture use and travel by cattle. In all instances, rate of decline was best fit with exponential decay functions (Figs. 2 and 4).

When estimating the proportion of pastures visited by cattle as GPS recording intervals were expanded, measures declined more rapidly with our point technique than with the line method (Fig. 2). The intention with the line method was to capture potential travel corridors between coordinates, and indeed the line technique inflated measures of resource use in all instances. With our 5- and 10-min recording intervals, the line method expanded measures of pasture use to $36.3 \pm 0.34 \%$ and $35.5 \pm 0.14 \%$, respectively (Fig. 2). Cattle that were active or grazing in this study moved at about $9.8 \pm 0.34 \mathrm{~m} \cdot \mathrm{min}^{-1}$. That velocity would likely find a cow within a single $100 \times$ $100 \mathrm{~m}$ pixel over a 5 -min and possibly a 10-min sampling interval. Thus, an argument that the inflationary component added by the line method was most likely valid data, could be strongly supported at the 5 -min scale and possibly with the $10-$ min interval. Indeed, only $8 \%$ of our observations at the 10 min interval were interpolated pixels that did not contain coordinates at the 5-min interval (Fig. 3).

Longer GPS integration intervals increasingly misrepresented travel corridors and, in some instances, suggested cattle escaped pasture boundaries or crossed barriers such as interior fences or cliffs. Indeed, in our most extreme case (1440-min or once-daily GPS recording interval), spatial error approached $80 \%$ (Fig. 3). If travel corridors are a critical concern, longer GPS sampling intervals and the line technique will likely lead to seriously flawed interpretations. Similarly, if measures of travel distances are important (Brosh et al. 2006), then we recommend the most frequent GPS sampling interval possible. These findings parallel those of Reynolds and Laundré (1990), who suggested maximization of information from radiotelemetry studies should be accomplished with a short sampling interval to satisfy more precise objectives. Other methods of analyses and measures of pasture use, such as distance from water or a critical resource (Bailey and Welling 1999; Ganskopp 2001) are less sensitive to longer GPS sampling intervals than the metrics evaluated in this study.

If equipment constraints mandate use of an infrequent GPS recording regime, our twice daily schedule showed an animal's relationship to critical resources might be biased if positions are acquired at the same time each day. Such a regime might suggest a biased reliance on water, shade, escape cover, and/or bedding areas if recording schedules were synchronized with the animal's daily routine. Thus, a scheduling algorithm that collected data at random times might provide a more accurate assessment of habitat use over the long run.

Finally, the consequence of selecting high-frequency GPS integration intervals still involves a tradeoff between high data resolution, battery life, and onboard memory. Recent advances in memory capacity now make it possible to store animal movements at 1-sec intervals for up to a week. With additional expense, one can include a radio link to remotely download collar data (Clark et al. 2006). This alleviates memory constraints, but battery longevity remains an issue. Battery drain increases rapidly as GPS integration intervals are shortened, and battery life is likely the limiting factor for extremely highfrequency regimes (Clark et al. 2006). This might not be an issue with domestic stock if one can capture animals and install fresh batteries, but the problem persists with studies of free-roaming wildlife. Ultimately, decisions regarding an appropriate GPS sampling frequency will depend on the specific objectives of one's research, the range and velocity of movement by the animal, and the resolution of one's habitat maps.

\section{MANAGEMENT IMPLICATIONS}

Although GPS collars are well proven tools for quantifying resource use, activity patterns, and travels of free-ranging animals, researchers must still address the quandary of memory 
and battery constraints specific to their instruments. An expansion of our most frequent recording interval of 5 min to 10 min could extend a study duration from 15 to $30 \mathrm{~d}$ without exhausting batteries. Misrepresentations of areas occupied would simultaneously increase from about $7 \%$ to $10 \%$, respectively, an error rate that could likely be tolerated in many instances. Successive expansions to 20 and $40 \mathrm{~min}$ recording intervals could extend projects even more, but increase spatial error rates to about $23 \%$ and $33 \%$, respectively. One might or might not feel comfortable making assumptions or management recommendations with data of that quality.

Extended studies are still needed, however, to capture seasonal or even annual distribution patterns of animals, especially in wildlife applications. Extremely infrequent recording regimes, such as a once-daily sampling interval, can introduce bias into one's interpretations about an animal's relationship to critical resources such as water, escape cover, or shade if the subject displays time-dependent movement patterns. If sampling at this scale is mandated, data acquisition at random times might provide better metrics of an animal's relationship to critical resources. One should not, however, make assumptions about an animal's use of resources along straight line courses between distant points or assume that such measures are accurate depictions of travel distances. These findings should help those using or considering use of GPS collars select sampling intervals that will maximize information returns and minimize spatial error.

\section{LITERATURE CITED}

Agouridis, C. T., T. S. Stombaugh, S. R. Workman, B. K. Koostra, D. R. Edwards, and E. S. Vanzant. 2004. Suitability of a GPS collar for grazing studies. Transactions-American Society of Agricultural Engineers 47:1321-1329.
Bailey, D. W., H. C. VanWagoner, and R. Weinmeister. 2006. Individual animal selection has the potential to improve uniformity of grazing on foothill rangeland. Rangeland Ecology \& Management 59:351-358.

Balley, D. W., AND G. R. Welling. 1999. Modification of cattle grazing distribution with dehydrated molasses supplement. Journal of Range Management 52:575-582.

Brosh, A., Z. Henkin, E. D. Ungar, A. Dolev, A. Orlov, Y. Yehuda, and Y. Aharoni. 2006. Energy cost of cows' grazing activity: use of the heart rate method and the global positioning system for direct field estimation. Journal of Animal Science 84:1951-1967.

Clark, P. E., D. E. Johnson, M. A. Kniep, P. Jermann, B. Huttash, A. Wood, M. Johnson, C. McGillivan, and K. Titus. 2006. An advanced, low-cost, GPSbased animal tracking system. Rangeland Ecology \& Management 59: 334-340.

Ganskopp, D. C. 2001. Manipulating cattle distribution with salt and water in large arid-land pastures: a GPS/GIS assessment. Applied Animal Behaviour Science 73:251-262.

Marquardt, D. W. 1963. An algorithm for least squares estimation of non-linear parameters. Journal of the Society of Industrial and Applied Mathematics 11:431-441.

Mourão, G., And I. M. MedRI. 2002. A new way of using inexpensive large-scale assembled GPS to monitor giant anteaters in short time intervals. Wildlife Society Bulletin 30:1029-1032.

ReYnolds, T. D., AND J. W. LAUndRÉ. 1990. Time intervals for estimating pronghorn and coyote home ranges and daily movements. Journal of Wildlife Management 54:316-322.

Rodgers, A. R., AND P. Anson. 1994. Animal-borne GPS: tracking the habitat. GPS World 5:20-32.

Rodgers, A. R., R. S. Rempel, and K. F. Abraham. 1996. A GPS-based telemetry system. Wildlife Society Bulletin 24:559-566.

Stuth, J. W. 1991. Foraging behavior. In: R. K. Heitschmidt and J. W. Stuth [eds.]. Grazing management: an ecological perspective. Portland, OR, USA: Timber Press. p. 65-83.

Ungar, E. D., Z. Henkin, M. Gutman, A. Dolve, A. Genizi, and D. Ganskopp. 2005. Inference of animal activity from GPS collar data on free-ranging cattle. Rangeland Ecology \& Management 58:256-266. 